\title{
Airway Management for Emergent Surgeries during COVID-19 Pandemic
}

\author{
Muhammad Zahoor and Ihtisham UI Haq \\ Department of Anesthesia, Armed Forces Hospital, Jizan, Kingdom of Saudi Arabia
}

\begin{abstract}
Aerosol generating procedures (AGPs) performed in the operating room during general anesthesia and surgery can contaminate the operation room environment putting the anesthetist, surgeons and paramedical staff at risk of infection with the novel severe acute respiratory syndrome coronavirus 2 (SARS-CoV-2) infection. The polymerase chain reaction (PCR) test for SARS-CoV-2 has low sensitivity and is time-bound. Emergent surgical cases might not give ample time for SARS-CoV-2 evaluation. These issues have called for adaptation of an anesthesia technique that can ensure safety measures regarding airway management in emergent cases; and can limit the chances of viral spread in case the patient is a carrier of coronavirus disease 2019 (COVID-19). In this communication, we summarised the modifications required in anesthesia technique during intubation and extubation of a patient's airway that would decrease the risk of virus transmission to the operation theatre staff.
\end{abstract}

Key Words: COVID-19, SARS-CoV-2, Emergent surgeries, Anesthesia technique.

How to cite this article: Zahoor M, Haq IU. Airway Management for Emergent Surgeries during COVID-19 Pandemic. J Coll Physicians Surg Pak 2021; 31(JCPSPCR):CR35-CR37.

The coronavirus disease 2019 (COVID-19) is a highly contagious viral infection, which in certain cases can lead to severe acute respiratory syndrome and even death. Earlier cases of the disease were diagnosed in Wuhan city of China in December 2019. The disease is caused by severe acute respiratory syndrome coronavirus 2 (SARS-CoV-2). The infected individuals spread the disease through micro-droplets when they breathe, cough or sneeze. In medical practice, the aerosol generating procedures (AGPs) are most likely to spread the disease. ${ }^{1}$ Due to this fact, any AGP performed in the operating room will put the anesthetist and paramedical staff at greater risk. Face mask ventilation, intubation, extubation, tracheostomy and laparoscopic surgeries are also high risk procedures. Emergent surgeries being norm in operating rooms are fast tracked with appropriate investigation and preparation. With new risk factors tied with emergent cases during this pandemic, safety of operating room staff, surgeons and anaesthetists can be compromised, if urgency of the case surpasses the need for PCR testing of the patient for SARS-CoV-2 infection.

Correspondence to: Dr. Muhammad Zahoor, Department of Anesthesia, Armed Forces Hospital, Jizan, Kingdom of Saudi Arabia

E-mail: drumarzahoor@hotmail.com

Received: September 01, 2020; Revised: September 04, 2020; Accepted: November 26, 2020

DOI: https://doi.org/10.29271/jcpsp.2021.JCPSPCR.CR35
Keeping in mind the above scenario, a pressing need has arisen for a comprehensive protocol regarding airway management, which can minimise the risk of infection to the caregivers in operating room, while maintaining high standard and quality of anaesthesia delivered to the patient. The patients, who are received for the procedure in operating room, should be wearing a surgical mask. Wherever possible the operation theatres should be converted into negative pressure rooms. ${ }^{2}$

Most of the published literature addresses different aspects of general anesthesia and airway management in order to avoid spread of COVID-19 from potential patient source. The aim of this communication is to bring together these guidelines, so that physicians dealing with the patient airway can adapt a safer comprehensive technique.

\section{Modification in induction technique for intubation:}

After going through published literature, we have concluded that following steps can minimise the aerosol generation at the time of tracheal intubation. The technique emphasis is on swift and smooth endotracheal tube placement. ${ }^{3,4}$

At the time of induction of general anesthesia, bag mask ventilation before intubation is bestavoided.

Patient should be placed in $30^{\circ}$ head-up position and pre-oxygenated with $100 \%$ oxygen.

A tight seal should be made with the mask in order to prevent anyleak.

Rapid sequence induction with succinylcholine is the method of 
choice unless there is a contra-indication to its use where it can be replaced with rocuronium, $1.2 \mathrm{mg} / \mathrm{kg}^{5}{ }^{5}$

Cricoid pressure is required in case patient is expected to be full stomach.

In case ventilation is required to achieve sufficient depth of anesthesia, low tidal volumeshould be used.

Two-hand technique can help prevent the leak from sides of face mask.

It is better to use video laryngoscope with a separate screen, so that the operator does not come close to the patient airway.

As soon as the endotracheal tube is in place, cuff should be inflated before connecting with the anesthesia circuit.

Attach end tidal carbon dioxide $\left(\mathrm{ETCO}_{2}\right)$ to the circuit to ensure the correct position of the endotracheal tube. Auscultating the chest may either be hazardous as it will bring the operator close to the patient or might not be possible if the anesthetist is wearing purified air pressure respirator (PARP). In such instance, it is better to rely on the equal chest rise and end tidal carbon dioxide $\left(\mathrm{ETCO}_{2}\right)$.

It is better to secure airway with endotracheal tube rather than supraglottic airway (SGA) devices, because of risk of air leak around the SGA device.

The guidelines of dealing with the difficult airway remain the same. However, awake fiberoptic intubation cannot be the first choice because of risk of aerosol generation. ${ }^{6}$ The local anesthetic nebulisation and spray as you go technique can be replaced with nerve blocks to avoid coughing. The tracheal injection of the local anesthetic is best avoided.

\section{Modification in technique for extubation:}

In routine administration of general anesthesia, the endotracheal tube is removed from the trachea either by applying positive airway pressure or by applying negative suction pressure to the endotracheal tube. Either way, it can be a source of aerosol generation.

However, adherence to certain guidelines, as explained in various literatures, can limit the exposure of staff to the aerosols at the time of extubation. ${ }^{7}$

Wherever possible, extubation should be carried out in negative pressure room, limiting the number of personnel available in room to bare minimum and wearing personal protective equipment (PPE).

Patient should bekept at $30^{\circ}$ head-up position.

Bite block to be placed to avoid patient bucking on the tube.

Use of anti-sialagogue drug such as glycopyrrolate to dry secretions minimising the need for suctioning.

An anti-emetic drug be administered to reduce the chances of vomiting at the time of extubation.
The chances of post-extubation laryngospasm and coughing can be reduced with the use of lidocaine (intravenous, intracuff or tracheal application), dexmedetomidine, remifentanyl and fentanyl. ${ }^{8}$

If oropharyngeal suctioning is required, it should be done after placing a clear plastic shield over the patient face. ${ }^{9}$

Endotracheal tube should be removed at the end of expiration.

As the endotracheal tube is removed, the mask should be placed and held with both hands technique to avoid any As soon as patient has established a viable airway and breathing has become normal, anesthesia mask can be replaced with nasal cannula or facial oxygen mask.

Patient should be given a surgical mask to be worn over the nasal orfacial oxygen mask.

In the recovery room, patient should continue to wear the surgical mask with supplemental oxygen, if required. Procedures like nebulisation should be avoided as they tend to increase the risk of aerosol production.

In conclusion, the above modifications in anesthesia technique during intubation and extubation of a patient's airway are important to decrease the risk of virus transmission to the operation theatre staff during the current COVID-19 pandemic and should beadapted where appropriate.

\section{CONFLICT OF INTEREST:}

We have no affiliation with any organisation or entity with respect to any financial or non-financial interests, while in the preparation of this manuscript.

\section{AUTHORS' CONTRIBUTION:}

MZ, IUH: Conception, gathering of material and proofreading.

\section{REFERENCES}

1. Van Doremalen N, Bushmaker T, Morris DH, Holbrook MG, Gamble A, Williamson BN et al. Aerosol and surface stability of HCoV-19 (SARS-CoV-2) compared to SARSCoV-1. N Eng J Med 2020; 382(16):1564-7. DOI: 10.1056/ NEJMc2004973.

2. Wax RS, Chritian MD. Practical recommendations for critical care and anesthesiology teams caring for novel coronavirus (2019-nCoV) patients. Can J Anesth 2020; 67(5):568-76. doi: 10.1007/s12630-020-01591-x.

3. Ong $S$, Khee $\Pi$. Practical considerations in the anaesthetic management of patients during a COVID-19 epidemic. Anaesthesia 2020; 75(6):823-4. doi: 10.1111/anae.15053.

4. Kim HJ, Sangwook Ko J, Kim TY. Recommendations for anesthesia in patients suspected of COVID-19 coronavirus infection. Korean J Anesthesiol 2020; 73(2):89-91. doi.org/ 10.4097/kja.20110.

5. Cook TM, El-Boghdadly K, McGuire B, McNarry AF, Patel A, Higgs $A$. Consensus guidelines for managing the airway in patients with COVID-19: Guidelines from the difficult airway society, the association of anaesthetists the intensive care society, the faculty of intensive care medicine and the royal college of anaesthetists. Anaesthesia 2020; 75(6):785-99. 
doi: 10.1111/anae.15054.

6. David FD'Silva, Timothy J McCulloch, Jessica S Lim, Sanchia $S$ Smith, Carayannis D. Extubation of patients with COVID-19. Br J Anaesth Advance Access 2020; 125(1): e192-5. doi: 10.1016/j.bja.2020.03.016.

7. Tung A, Fergusson NA, Nicole NG, Viven HU, Dormuth C, Griesdale DEG. Medications to reduce emergence coughing after general anaesthesia with tracheal intubation: A systemic review and network meta-analysis. Br J Anaesth
2020; 124(4):480-95. doi: 10.1016/j.bja.2019.12.041.

8. Matava CT, Julie Yu, Denning S. Clear plastic drapes may be effective at limiting aerosolisation and droplet spray during extubation: Implications for COVID 19. Can J Anesth 2020; 67(7):902-4. doi: 10.1007/s12630-020-01649-w.

9. Wong J, Goh QY, Tan Z, Lie SA, Tay Y C, Ng SY, et al. Preparing for a COVID-19 pandemic: A review of operating room outbreak response measures in a large tertiary hospital in Singapore. Can J Anesth 2020; 67(6):732-45. doi: 10.1007/s12630-020-01620-9. 